\title{
A high-sensitivity $\mathrm{OH}$ 5-cm line survey in late-type stars ${ }^{\star}$
}

\author{
J.-F. Desmurs ${ }^{1}$, A. Baudry ${ }^{2}$, P. Sivagnanam ${ }^{3}$, and C. Henkel ${ }^{4}$ \\ 1 Observatorio Astronómico Nacional (IGN), Apartado 1143, 28800 Alcalá de Henares, Spain \\ 2 Observatoire de l'Université de Bordeaux, URA 352 du CNRS, BP 89, 33270 Floirac, France \\ 3 Observatoire de Paris, 5 place Jules Janssen, 92195 Meudon Cedex, France \\ ${ }^{4}$ Max-Planck-Institut für Radioastronomie, Auf dem Hügel 69, 53121 Bonn, Germany
}

Received 7 May 2002 / Accepted 27 August 2002

\begin{abstract}
We have undertaken a comprehensive search for 5-cm excited $\mathrm{OH}$ maser emission from evolved stars representative of various stages of late stellar evolution. Observed sources were selected from known $18-\mathrm{cm} \mathrm{OH}$ sources. This survey was conducted with the 100-m Effelsberg telescope to achieve high signal to noise ratio observations and a sensitivity limit of about 0.05 to $0.1 \mathrm{Jy}$. A total of 65 stellar sources were searched for both main line and satellite line emission. We confirm the previous detection of $5 \mathrm{~cm} \mathrm{OH}$ in $\mathrm{Vy}$ 2-2, do not confirm emission from NML-Cyg and do not report any other new detection within the above sensitivity limit.

Implications of these results on the pumping mechanism of the $\mathrm{OH}$ radical in circumstellar envelopes are briefly discussed.
\end{abstract}

Key words. masers - stars: AGB and post-AGB - radio lines: stars

\section{Introduction}

Emission from the first two rotationally excited states of $\mathrm{OH}$ was first discovered by Zuckerman et al. (1968) and Yen et al. (1969) for the ${ }^{2} \Pi_{1 / 2}, J=1 / 2$ and ${ }^{2} \Pi_{3 / 2}, J=5 / 2$ states, respectively. The ${ }^{2} \Pi_{3 / 2}, J=5 / 2$ state of $\mathrm{OH}$ lies immediately above the ground-state and gives rise to four hyperfine transitions, with the $F=3-3$ and 2-2 main lines at 6035.092 and $6030.747 \mathrm{MHz}$ and the $F=3-2$ and 2-3 satellite lines at 6049.084 and $6016.746 \mathrm{MHz}$, respectively (Fig. 1). The theoretical treatment of $\mathrm{OH}$ excitation in star-forming regions has progressed significantly in recent years (see Cesaroni \& Walmsley 1991; Gray et al. 1992; Pavlakis \& Kylafis 1996), and good predictions of relative $\mathrm{OH}$ line intensities can be made on the basis of these models, which show the importance of multi-line studies. In the circumstellar environment of latetype stars the model developed by Elitzur et al. (1976) successfully explains the excitation of strong $1612 \mathrm{MHz}$ emission. This results from a cascade of the $\mathrm{OH}$ population down to the $J=1 / 2$ and $3 / 2$ states after far infrared photons at 34.6 micron and 53.3 micron (see Fig. 1) have excited the $\mathrm{OH}$ to the ${ }^{2} \Pi_{1 / 2}, J=5 / 2$ and $3 / 2$ states. There are enough far infrared photons to excite the $1612 \mathrm{MHz}$ line (e.g. Epchtein et al. 1980). However, it is only recently that the direct detection of the 34.6 microns absorption line has been reported with the ISO telescope toward IRC+10420 (see Sylvester et al. 1997).

Send offprint requests to: J.-F. Desmurs, e-mail: desmurs@oan.es

* Table 1 is also available in electronic form at the CDS via anonymous ftp to cdsarc.u-strasbg.fr $(130.79 .128 .5)$ or via http://cdsweb.u-strasbg.fr/cgi-bin/qcat?J/A+A/394/975
Besides the conspicuous $1612 \mathrm{MHz}$ line emission, $18 \mathrm{~cm}$ main line emission is often observed in late-type stars. Conditions for this emission are carefully investigated in the work of Collison \& Nedoluha $(1994,1995)$ and we discuss later in this work the implication of their excitation mechanism for the $J=5 / 2$ state of $\mathrm{OH}$.

The main goal of the present observations was to survey the $5 \mathrm{~cm} \Lambda$ doublet lines of $\mathrm{OH}$ in a number of stars ranging from typical Miras to $\mathrm{OH} / \mathrm{IR}$ objects or pre-planetary nebulae. These stars sample various late stages of stellar evolution. In addition, observations of the ${ }^{2} \Pi_{3 / 2}, J=5 / 2$ state lying immediately above the ground-state provide a critical test for $\mathrm{OH}$ excitation models.

These observations and our results obtained in 65 sources are presented in Sects. 2 and 3. Some $\mathrm{OH}$ properties of selected stars are also presented in Sect. 3. In Sect. 4 we discuss stellar $\mathrm{OH}$ pumping schemes and variability of $\mathrm{OH}$ main line emission sources in the light of our results.

\section{Observations}

Observations were made with the 100-m antenna at Effelsberg ${ }^{1}$ in December 1999. We used a cooled HEMT dual-channel receiver connected to only one sense of polarization, Left Circular Polarization (LCP). The system temperature was $\approx 60 \mathrm{~K}\left(T_{\mathrm{mb}}\right)$ including ground pick up and sky noise. We

\footnotetext{
1 The 100-m telescope at Effelsberg is operated by the Max-PlanckInstitut für Radioastronomie (MPIfR) on behalf of the Max-PlanckGesellschaft (MPG).
} 


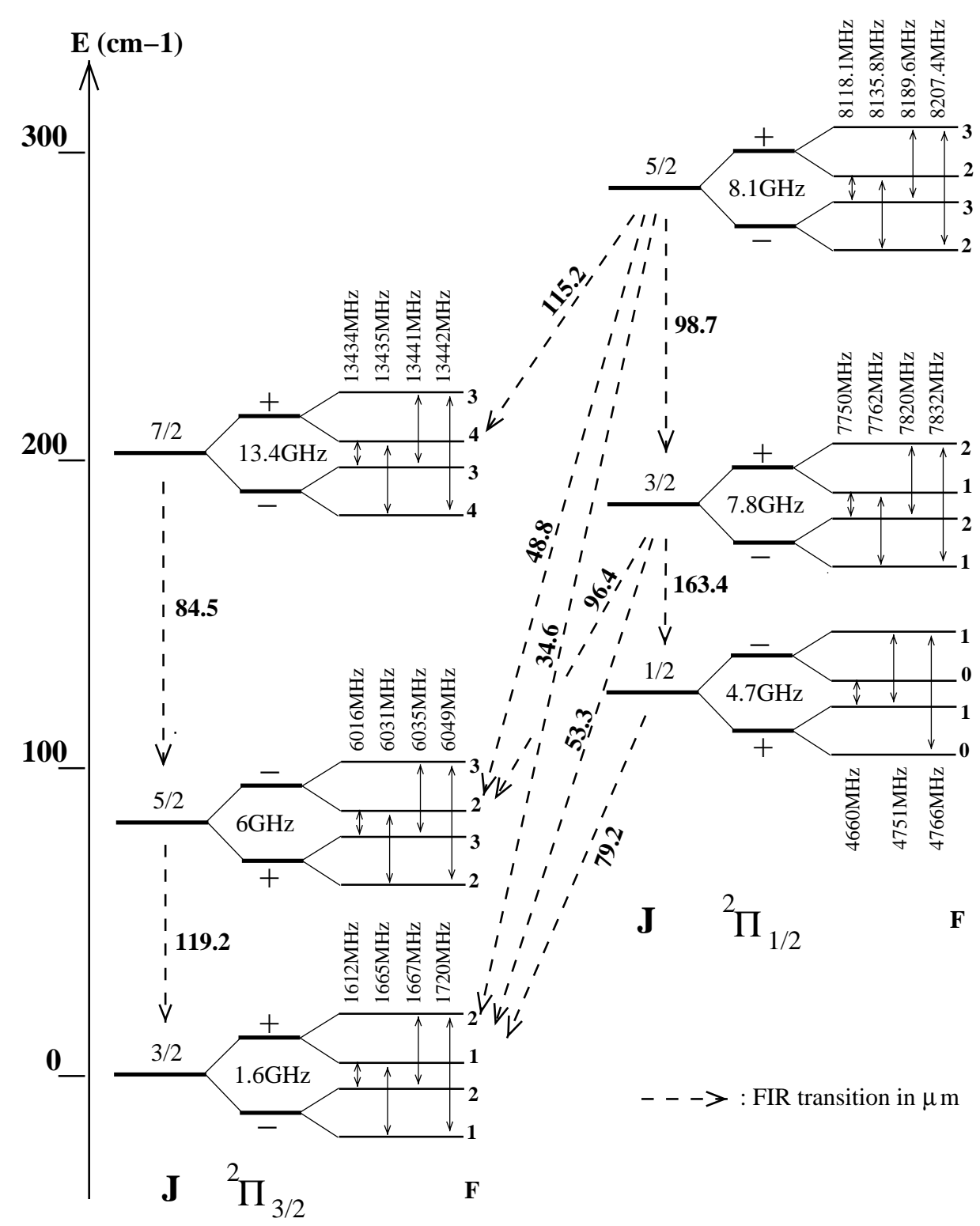

Fig. 1. The energy level diagram for the ${ }^{2} \Pi_{3 / 2}$ and ${ }^{2} \Pi_{1 / 2}$ ladders of OH. $\Lambda$ doubling (not to scale) and parities are shown in each case. Transitions between the $F=3$ and 2 hyperfine levels, for ${ }^{2} \Pi_{3 / 2}, J=5 / 2$, give rise to the four $6 \mathrm{GHz}$ lines.

used a position switching observing mode with the reference position offset by $600^{\prime \prime}$ in longitude from the source position (the half-power beamwidth of the telescope at $6 \mathrm{GHz}$ is $\left.130^{\prime \prime}\right)$. The new auto-correlator AK90 was split into 2 bands of $20 \mathrm{MHz}$ each thus allowing us to simultaneously observe the 2 main lines and the two satellites lines of the $J=5 / 2$ state. There were 4096 channels per band giving a channel separation of $4.9 \mathrm{kHz}$ and thus an effective spectral velocity resolution of $0.29 \mathrm{~km} \mathrm{~s}^{-1}$. Proper functioning of the system was checked by observations of the strong $5 \mathrm{~cm} \mathrm{OH}$ emission from the two compact HII regions W3(OH) and ON1 (see Table 1).

Calibration of the data followed the procedure used in the $6 \mathrm{GHz}$ survey of star-forming regions made by Baudry et al. (1997). OH spectra were calibrated in terms of the noise source coupled to one polarization channel and the flux density scale was determined by observations of NGC 7027 (Ott et al. 1994). The noise tube was calibrated in Jy assuming that the $6 \mathrm{GHz}$ flux density of NGC 7027 was $5.9 \mathrm{Jy}$. We estimate that the flux density scale uncertainty is within $10 \%$. All spectra were calibrated in terms of single polarization flux densities. This is one-half of the two polarization flux density. For possible $5 \mathrm{~cm}$ radio interference, we proceeded as in Baudry et al. (1997).

Our input catalog is listed in Table 1. It is based on $18 \mathrm{~cm} \mathrm{OH}$ data. We selected sources which clearly exhibit the $1612 \mathrm{MHz}$ satellite line and/or the 1665/1667 main lines. By these means we obtained targets with noticeable amounts of $\mathrm{OH}$ molecules and IR photons, that are not excessively distant in order to be detected.

The sources are essentially $\mathrm{OH}$ Miras with thin or moderately thick envelopes, and thick $\mathrm{OH} / \mathrm{IR}$ objects. Bright Miras with both satellite and main lines were selected, from the Sivagnanam et al. (1988) comprehensive OH survey of the $1-\mathrm{kpc}$ solar neighborhood. Most of them are also known as $22 \mathrm{GHz}$ water maser sources. From the David et al. (1993) 
Table 1. Observations of the $6 \mathrm{GHz} \mathrm{OH}$ maser transitions in AGB stars.

\begin{tabular}{|c|c|c|c|c|c|c|}
\hline \multirow[t]{3}{*}{ IRAS Source } & \multirow[t]{3}{*}{ Other Name } & \multirow[t]{3}{*}{ Type $^{1}$} & \multicolumn{2}{|c|}{$\begin{array}{c}\text { Observed Coordinates } \\
\mathrm{J} 2000.0^{3}\end{array}$} & \multirow{3}{*}{$\begin{array}{c}\text { Observed } \\
\text { Velocity } \\
\text { Range, LSR } \\
\left(\mathrm{km} \mathrm{s}^{-1}\right) \\
\end{array}$} & \multirow{3}{*}{$\begin{array}{c}\text { Sensitivity }^{2}(\mathrm{Jy}) \\
(\text { at } 3 \sigma) \\
6 \mathrm{GHz} \\
\mathrm{LCP}\end{array}$} \\
\hline & & & RA & Dec & & \\
\hline & & & $\mathrm{h} \mathrm{m} \mathrm{s}$ & $\circ \prime \prime$ & & \\
\hline $02232+6138$ & $\mathrm{~W} 3(\mathrm{OH})$ & UCHII & $2: 27: 03.866$ & $+61: 52: 24.82$ & $-939 /+998,(-45.0)$ & $\mathrm{E}$ \\
\hline $20081+3122$ & ON1 & UCHII & 20:10:09.073 & $+31: 31: 34.40$ & $-894 / 1043,(+00.0)$ & $\mathrm{E}$ \\
\hline $00007+5524$ & Y Cas & Mira main & $00: 03: 21.605$ & $+55: 40: 48.21$ & $-911 / 1026,(-17.0)$ & 0.08 \\
\hline $01037+1219$ & IRC +10011 & $\mathrm{OH} / \mathrm{IR}$ Moderate & 01:06:25.965 & $+12: 35: 53.07$ & $-886 / 1051,(+08.0)$ & 0.07 \\
\hline $02168-0312$ & OMI Cet & Mira main & 02:19:20.679 & $-02: 58: 39.21$ & $-848 / 1089,(+46.0)$ & 0.06 \\
\hline $02192+5821$ & S Per & SG & $02: 22: 51.689$ & $+58: 35: 11.76$ & $-932 / 1005,(-38.0)$ & 0.08 \\
\hline $02251+5102$ & RR Per & Mira main & $02: 28: 29.318$ & $+51: 16: 17.60$ & $-894 / 1043,(+00.0)$ & 0.08 \\
\hline $02420+1206$ & RU Ari & Mira sat & $02: 44: 45.184$ & $+12: 19: 08.15$ & $-874 / 1063,(+20.0)$ & 0.07 \\
\hline $03206+6521$ & $\mathrm{OH} 138.0+7.2$ & $\mathrm{OH} / \mathrm{IR}$ Moderate & $03: 25: 08.552$ & $+65: 32: 05.42$ & $-931 / 1006,(-37.5)$ & 0.08 \\
\hline $03293+6010$ & $\mathrm{OH} 141.0+3.5$ & $\mathrm{OH} / \mathrm{IR}$ Moderate & $03: 33: 30.528$ & $+60: 20: 09.10$ & $-951 / 986,(-57.2)$ & 0.07 \\
\hline $03507+1115$ & IK Tau & Mira sat & $03: 53: 28.983$ & $+11: 24: 20.02$ & $-861 / 1076,(+33.0)$ & 0.05 \\
\hline $05073+5248$ & NV Aur & $\mathrm{OH} / \mathrm{IR}$ Moderate & 05:11:19.747 & $+52: 52: 27.79$ & $-891 / 1046,(+02.9)$ & 0.08 \\
\hline $05131+4530$ & RAFGL 712 & $\mathrm{OH} / \mathrm{IR}$ Moderate & $05: 16: 47.103$ & $+45: 34: 03.76$ & $-926 / 1011,(-32.3)$ & 0.09 \\
\hline $05528+2010$ & U Ori & Mira sat & $05: 55: 49.264$ & $+20: 10: 30.77$ & $-933 / 1004,(-39.5)$ & 0.04 \\
\hline $05559+7430$ & V Cam & Mira main & 06:02:32.779 & $+74: 30: 27.26$ & $-887 / 1050,(+06.5)$ & 0.07 \\
\hline $06297+4045$ & OH $174.7+13.5$ & $\mathrm{OH} / \mathrm{IR}$ Moderate & 06:33:14.921 & $+40: 42: 49.71$ & $-910 / 1027,(-16.3)$ & 0.08 \\
\hline $06363+5954$ & U Lyn & Mira main & $06: 40: 46.320$ & $+59: 52: 00.23$ & $-904 / 1033,(-10.0)$ & 0.07 \\
\hline $06500+0829$ & GX Mon & Mira sat & $06: 52: 42.408$ & $+08: 25: 23.33$ & $-905 / 1032,(-11.0)$ & 0.06 \\
\hline $07209-2540$ & VY Cma & SG & 07:22:58.17 & $-25: 46: 02.95$ & $-872 / 1065,(+22.0)$ & 0.07 \\
\hline $07399-1435$ & $\mathrm{OH} 231.8+4.2$ & PPN & $07: 42: 16.738$ & $-14: 42: 14.04$ & $-878 / 1059,(+16.0)$ & 0.06 \\
\hline $07422+3054$ & AU Gem & Mira Main & $07: 45: 27.590$ & $+30: 46: 43.11$ & $-890 / 1047,(+04.0)$ & 0.04 \\
\hline $07585-1242$ & U Pup & Mira sat & 08:00:50.596 & $-12: 50: 31.88$ & $-912 / 1025,(-18.0)$ & 0.04 \\
\hline $08005+2356$ & & $\mathrm{OH} / \mathrm{IR}$ Late & 08:02:59.186 & $+23: 45: 33.23$ & $-888 / 1049,(+05.7)$ & 0.07 \\
\hline $08357-1013$ & $\mathrm{OH} 235.3+18.1$ & $\mathrm{OH} / \mathrm{IR}$ Moderate & 08:38:08.805 & $-10: 24: 16.95$ & $-894 / 1043,(+00.0)$ & 0.07 \\
\hline $09425+3444$ & R Lmi & Mira main & $09: 45: 34.314$ & $+34: 30: 42.91$ & $-894 / 1043,(+00.0)$ & 0.14 \\
\hline $09448+1139$ & R Leo & Mira main & $09: 47: 33.446$ & $+11: 25: 43.52$ & $-893 / 1044,(+01.0)$ & 0.05 \\
\hline $10580-1803$ & $\mathrm{R} \mathrm{Crt}$ & SR & 11:00:33.822 & $-18: 19: 29.54$ & $-884 / 1053,(+10.0)$ & 0.05 \\
\hline $12562+2324$ & T Com & Mira sat & $12: 58: 38.600$ & $+23: 08: 23.03$ & $-879 / 1058,(+15.0)$ & 0.07 \\
\hline $13001+0527$ & RT Vir & SR & 13:02:37.908 & $+05: 11: 08.56$ & $-876 / 1061,(+18.0)$ & 0.09 \\
\hline $14247+0454$ & RS Vir & Mira sat & $14: 27: 16.393$ & $+04: 40: 40.38$ & $-894 / 1043,(+00.0)$ & 0.08 \\
\hline $15193+3132$ & S Crb & Mira main & $15: 21: 24.366$ & $+31: 22: 02.05$ & $-894 / 1043,(+00.0)$ & 0.07 \\
\hline $15255+1944$ & WX Ser & Mira sat & $15: 27: 47.009$ & $+19: 34: 02.33$ & $-888 / 1049,(+06.0)$ & 0.08 \\
\hline $16235+1900$ & U Her & Mira main & $16: 25: 47.411$ & $+18: 53: 32.98$ & $-907 / 1030,(-13.0)$ & 0.05 \\
\hline $18006-1734$ & GLMP 704 & $\mathrm{OH} / \mathrm{IR}$ Late & 18:03:36.581 & $-17: 34: 00.59$ & $-870 / 1067,(+23.9)$ & 0.10 \\
\hline $18071-1727$ & OH $12.8+.9$ & $\mathrm{OH} / \mathrm{IR}$ Late & 18:10:05.813 & $-17: 26: 35.22$ & $-868 / 1069,(+25.9)$ & 0.09 \\
\hline $18081-0338$ & & OH/IR Thick & $18: 10: 49.074$ & $-03: 38: 14.46$ & $-889 / 1049,(+05.6)$ & 0.08 \\
\hline $18100-1915$ & GLMP 740 & $\mathrm{OH} / \mathrm{IR}$ Thick & $18: 13: 02.725$ & $-19: 14: 20.42$ & $-876 / 1061,(+17.6)$ & 0.09 \\
\hline $18107-0710$ & & OH/IR Thick & $18: 13: 29.720$ & $-07: 09: 48.92$ & $-876 / 1061,(+17.9)$ & 0.08 \\
\hline $18135-1456$ & $\mathrm{OH} 15.7+.8$ & $\mathrm{OH} / \mathrm{IR}$ Late & $18: 16: 26.004$ & $-14: 55: 13.43$ & $-895 / 1042,(-01.1)$ & 0.09 \\
\hline
\end{tabular}

Nançay survey of $\mathrm{OH} / \mathrm{IR}$ objects, we retained bright $1612 \mathrm{MHz}$ sources with detected $1667 \mathrm{MHz}$ emission. Targets have been chosen in order to sample objects lying along the sequence drawn by evolved stars in the IRAS two-color diagram (see Fig. 2). They mostly belong to the AGB. This sequence of envelope thicknesses is thought to reflect various evolutionary stages, and a certain initial mass distribution. Given our selection criteria we favored the best candidate stars for showing $\mathrm{OH}$ maser emission i.e. the nearby $\mathrm{OH} / \mathrm{IR}$ stars with high luminosity and large amounts of dust and infrared photons.

We also included in our survey a few typical Semi-Regular variables, Proto-Planetary Nebulae (PPN), and Planetary Nebulae (PN), that are thought to be linked to the same evolutionary tracks. We added some red Supergiants (SG). 
Table 1. continued.

\begin{tabular}{|c|c|c|c|c|c|c|}
\hline \multirow[t]{3}{*}{ IRAS Source } & \multirow[t]{3}{*}{ Other Name } & \multirow[t]{3}{*}{ Type $^{1}$} & \multicolumn{2}{|c|}{$\begin{array}{c}\text { Observed Coordinates } \\
\mathrm{J} 2000.0^{3}\end{array}$} & \multirow{3}{*}{$\begin{array}{c}\text { Observed } \\
\text { Velocity } \\
\text { Range, LSR } \\
\left(\mathrm{km} \mathrm{s}^{-1}\right)\end{array}$} & \multirow{3}{*}{$\begin{array}{c}\text { Sensitivity }^{2}(\mathrm{Jy}) \\
\text { (at } 3 \sigma) \\
6 \mathrm{GHz} \\
\text { LCP }\end{array}$} \\
\hline & & & RA & Dec & & \\
\hline & & & $\mathrm{h} \mathrm{m} \mathrm{s}$ & 0,11 & & \\
\hline $18152-0919$ & OH $20.8+3.1$ & OH/IR Thick & $18: 17: 58.662$ & $-09: 18: 42.44$ & $-867 / 1070,(+26.7)$ & 0.08 \\
\hline $18198-1249$ & $\mathrm{OH} 18.3+.4$ & $\mathrm{OH} / \mathrm{IR}$ Late & $18: 22: 43.046$ & $-12: 47: 40.94$ & $-846 / 1091,(+48.0)$ & 0.10 \\
\hline $18262-0735$ & & $\mathrm{OH} / \mathrm{IR}$ Thick & $18: 28: 59.446$ & $-07: 33: 25.44$ & $-815 / 1122,(+78.9)$ & 0.08 \\
\hline $18268-1117$ & OH $20.4-.3$ & $\mathrm{OH} / \mathrm{IR}$ Thick & $18: 29: 35.755$ & $-11: 15: 53.97$ & $-852 / 1085,(+41.8)$ & 0.08 \\
\hline $18266-1239$ & V435 Sct & OH/IR Thick & $18: 29: 28.605$ & $-12: 37: 40.55$ & $-844 / 1093,(+50.0)$ & 0.08 \\
\hline $18348-0526$ & V437 Sct & OH/IR Thick & $18: 37: 31.986$ & $-05: 23: 59.35$ & $-867 / 1070,(+27.2)$ & 0.06 \\
\hline $18432-0149$ & V1360 Aql & $\mathrm{OH} / \mathrm{IR}$ Thick & $18: 45: 52.691$ & $-01: 46: 43.27$ & $-828 / 1109,(+66.2)$ & 0.08 \\
\hline $18460-0254$ & V1362 Aql & $\mathrm{OH} / \mathrm{IR}$ Late & 18:48:40.999 & $-02: 50: 22.29$ & $-795 / 1142,(+99.0)$ & 0.09 \\
\hline $18488-0107$ & V1363 Aql & $\mathrm{OH} / \mathrm{IR}$ Late & $18: 51: 25.772$ & $-01: 03: 54.48$ & $-818 / 1119,(+75.8)$ & 0.08 \\
\hline $18525+0210$ & & OH/IR Thick & $18: 55: 04.815$ & $+02: 14: 41.21$ & $-823 / 1113,(+70.2)$ & 0.07 \\
\hline $18549+0208$ & OH $35.6-.3$ & $\mathrm{OH} / \mathrm{IR}$ Thick & $18: 57: 26.573$ & $+02: 12: 11.24$ & $-816 / 1121,(+77.9)$ & 0.09 \\
\hline $18560+0638$ & V1366 Aql & $\mathrm{OH} / \mathrm{IR}$ Moderate & 18:58:30.142 & $+06: 42: 55.91$ & $-874 / 1063,(+19.7)$ & 0.10 \\
\hline $19039+0809$ & R Aql & Mira sat & 19:06:22.196 & $+08: 13: 48.16$ & $-846 / 1091,(+48.0)$ & 0.09 \\
\hline $19065+0832$ & $\mathrm{OH} 42.6+.0$ & $\mathrm{OH} / \mathrm{IR}$ Late & 19:08:58.368 & $+08: 37: 47.08$ & $-841 / 1096,(+53.1)$ & 0.07 \\
\hline $19071+0946$ & $\mathrm{OH} 43.8+.5$ & $\mathrm{OH} / \mathrm{IR}$ Thick & 19:09:31.065 & $+09: 51: 54.41$ & $-885 / 1052,(+09.1)$ & 0.12 \\
\hline $19161+2343$ & & $\mathrm{OH} / \mathrm{IR}$ Moderate & $19: 18: 14.537$ & $+23: 49: 26.23$ & $-865 / 1072,(+28.6)$ & 0.07 \\
\hline $19219+0947$ & Vy $2-2$ & $\mathrm{PN}$ & $19: 24: 22.078$ & $+09: 53: 55.82$ & $-956 / 981,(-44.3)$ & $\mathrm{E} / 0.02$ \\
\hline $19244+1115$ & V1302 Aql & $\mathrm{OH} / \mathrm{IR}$ Moderate & $19: 26: 47.588$ & $+11: 21: 14.77$ & $-849 / 1088,(+45.0)$ & 0.08 \\
\hline $19343+2926$ & M1-92 & PPN & $19: 36: 16.768$ & $+29: 32: 15.80$ & $-894 / 1043,(+0.00)$ & 0.24 \\
\hline $19352+2030$ & & OH/IR Thick & $19: 37: 23.395$ & $+20: 39: 21.86$ & $-889 / 1048,(+05.0)$ & 0.20 \\
\hline $20043+2653$ & GLMP 972 & OH/IR Thick & 20:06:22.890 & $+27: 02: 11.23$ & $-898 / 1039,(-04.6)$ & 0.08 \\
\hline $20047+1248$ & SY Aql & Mira sat & 20:07:05.694 & $+12: 57: 07.39$ & $-943 / 994,(-49.0)$ & 0.08 \\
\hline NML Cyg & & SG & $20: 46: 25.941$ & $+40: 06: 56.09$ & $-894 / 1043,(+00.0)$ & 0.02 \\
\hline $20491+4236$ & OH $83.4-.9$ & OH/IR Moderate & $20: 50: 57.766$ & $+42: 48: 04.31$ & $-932 / 1005,(-38.4)$ & 0.05 \\
\hline $21554+6204$ & GLMP 1048 & OH/IR Thick & $21: 56: 58.184$ & $+62: 18: 43.62$ & $-914 / 1022,(-20.6)$ & 0.06 \\
\hline $22177+5936$ & NSV 25875 & $\mathrm{OH} / \mathrm{IR}$ Moderate & $22: 19: 27.806$ & $+59: 51: 21.74$ & $-919 / 1017,(-25.6)$ & 0.07 \\
\hline $23041+1016$ & R Peg & Mira main & 23:06:38.829 & $+10: 32: 37.94$ & $-870 / 1067,(+24.0)$ & 0.09 \\
\hline $23558+5106$ & R Cas & Mira main & $23: 58: 24.683$ & $+51: 23: 18.18$ & $-870 / 1067,(+24.0)$ & 0.06 \\
\hline
\end{tabular}

${ }^{1}$ UCHII: Ultra Compact HII region, SG: Supergiante, SR: Semi Regular, PN: Planetary Nebulae, PPN: Proto Planetary Nebulae.

${ }^{2} \mathrm{E}=$ Emission; the upper limits correspond to $3 \sigma$.

${ }^{3}$ B1950 coordinates processed to J2000 within GILDAS package.

The latter are luminous massive stars that are not on the AGB, but which usually present somewhat similar circumstellar envelopes. In our input catalog are NML Cyg (SG) and Vy 2-2 (PN), for which tentative detections at $5 \mathrm{~cm}$ have been reported in the literature.

\section{Results}

\subsection{Previous surveys}

Previous attempts made to search for excited $\mathrm{OH}$ from circumstellar envelopes gave only negative or controversial results with the exception of one object. As far as we are aware only a few searches for $J=1 / 2$ and $5 / 2 \mathrm{OH}$ emission at 4.7 and $6 \mathrm{GHz}$ from stars have been undertaken (see Thacker et al. 1970; Zuckerman et al. 1972; Baudry 1974;
Claussen \& Fix 1981; Jewell et al. 1985). The latter work was the most sensitive search for excited $\mathrm{OH}$ from stars yet performed. Zuckerman et al. (1972) reported weak $6035 \mathrm{MHz}$ $\left({ }^{2} \Pi_{3 / 2}, J=5 / 2\right)$ emission from NML Cyg and Claussen \& Fix (1981) reported weak $4751 \mathrm{MHz}\left({ }^{2} \Pi_{1 / 2}, J=1 / 2\right)$ emission from AU Gem. However, both detections were not confirmed by Jewell et al. (1985). On the other hand, Jewell et al., reported weak $6035 \mathrm{MHz}$ maser emission from the planetary nebula Vy 2-2 appearing at the same velocity, $-62 \mathrm{~km} \mathrm{~s}^{-1}$, as the peak $1612 \mathrm{MHz}$ maser emission detected by Davis et al. (1979).

\subsection{New Effelsberg survey}

In Table 1, we list the 65 late type stars observed by us. For all sources, the velocity range of search for emission is given 


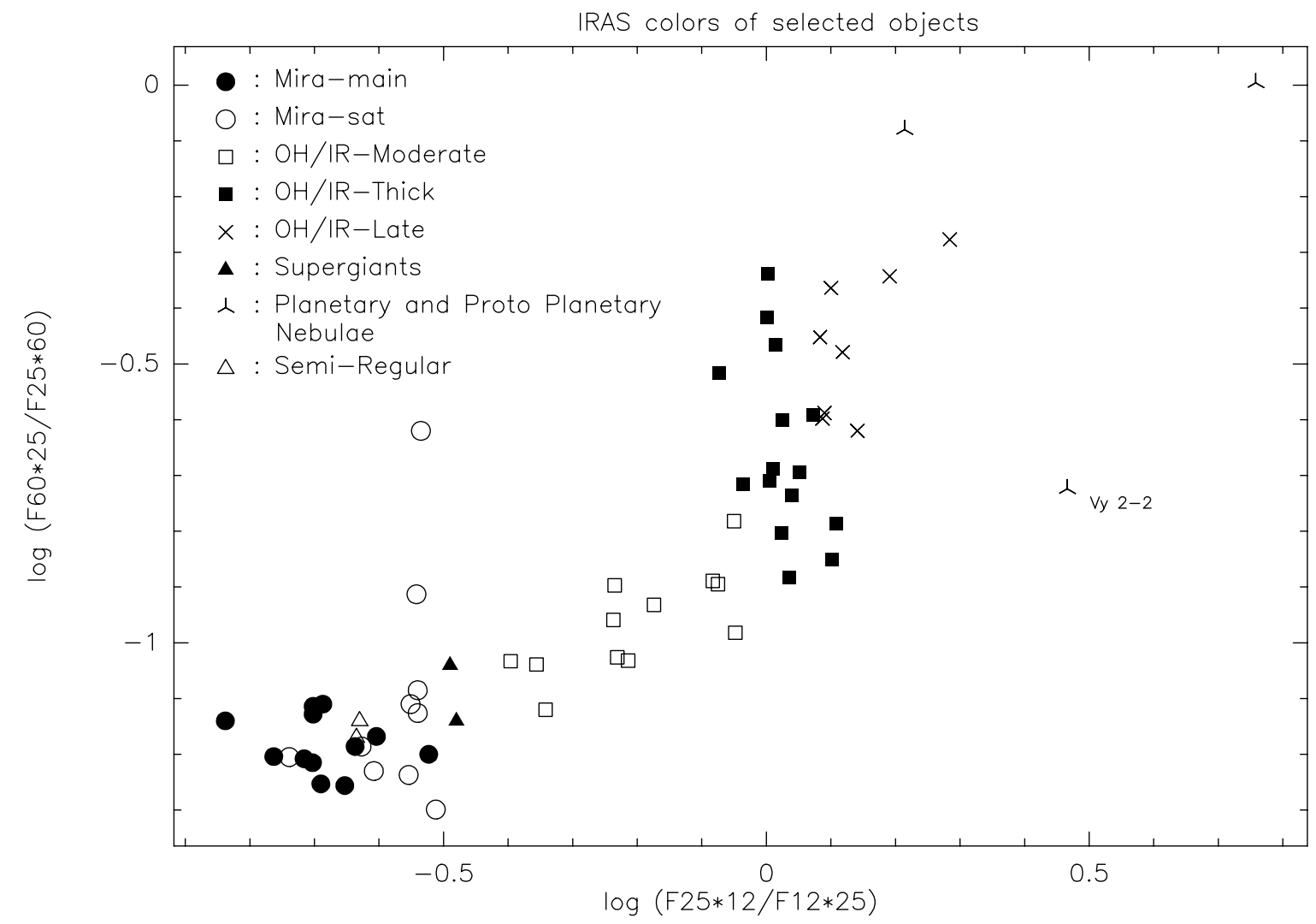

Fig. 2. Uncorrected IRAS colors from IRAS flux measurements available of the sources in our sample. (NML Cyg is not included as there is no IRAS measurement available.)

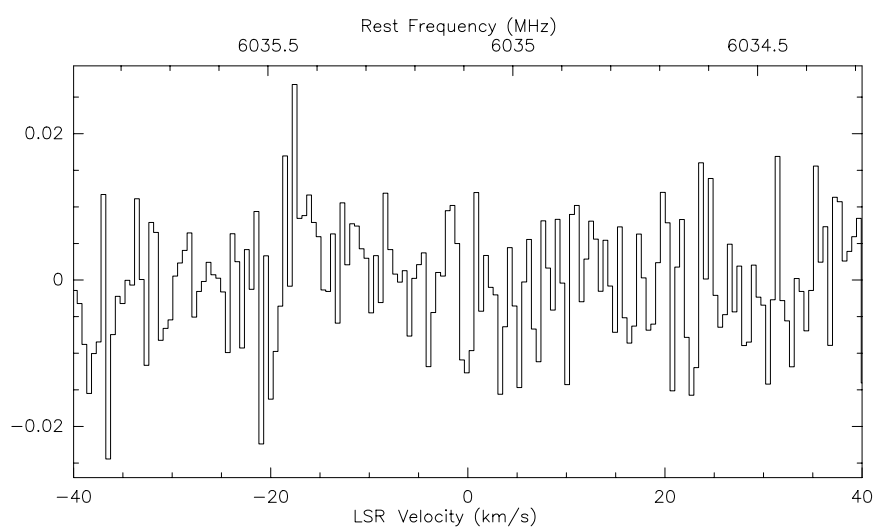

Fig. 3. The $6 \mathrm{GHz}$ spectra obtained in Dec 1999 for NML Cyg, the rms at $1 \sigma$ is $\sim 6 \mathrm{mJy}$ (LCP spectrum). No detection reported.

(with the systemic velocity in parenthesis), together with the sensitivity limit achieved in our new survey at $3 \sigma$. The average noise level reached in our survey is (at $3 \sigma$ with a channel width of $0.29 \mathrm{~km} \mathrm{~s}^{-1}$ ) around $80 \mathrm{mJy}$; in comparison, Jewell et al. (1985) have reached about $230 \mathrm{mJy}$ (with a channel width of $0.06 \mathrm{~km} \mathrm{~s}^{-1}$ ).

Of the 65 sources observed, no one exhibits a clear emission or absorption signal. There are however two sources with tentative detections, NML Cyg (see Fig. 3) and Vy 2-2 (Fig. 4). For NML Cyg, we reached the sensitivity of $20 \mathrm{mJy}$ (at $3 \sigma$ level) over the observing LSR velocity range. The $0.8 \mathrm{~K}$ (2.2Jy) signal reported by Zuckerman et al. (1972) and lying close

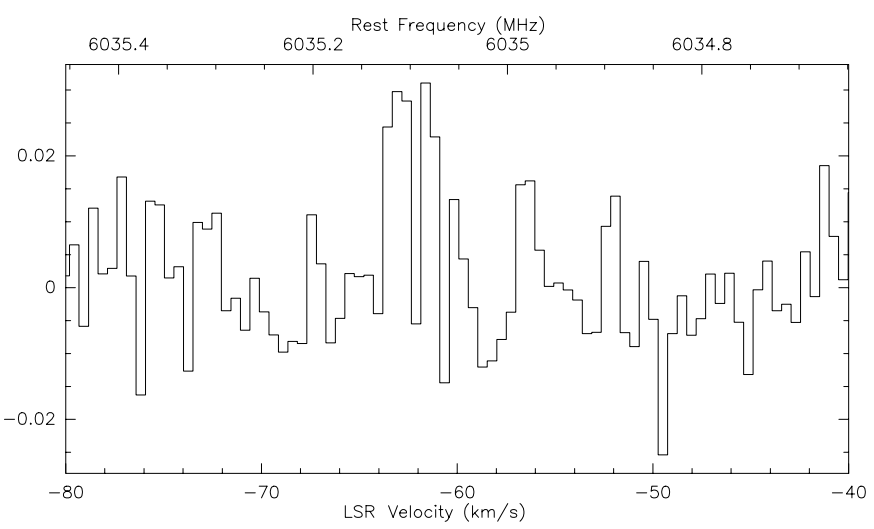

Fig. 4. $6035 \mathrm{MHz} \mathrm{OH}$ spectrum obtained in Dec. 1999 from Vy 2-2. The line intensity is in Jy for single polarization, the rms at $1 \sigma$ is $\sim 6 \mathrm{mJy}$.

to $+5 \mathrm{~km} \mathrm{~s}^{-1}$, would have been easily detected by us. However, we can not exclude that the emission varies with time. The tentative feature at about $-17 \mathrm{~km} \mathrm{~s}^{-1}$ (Fig. 4) is only detected at a $\sim 3 \sigma$ level and is therefore not convincing, but we note that $1612 \mathrm{MHz}$ line emission at $-18 \mathrm{~km} \mathrm{~s}^{-1}$ has been reported previously (see e.g. Engels 1979).

The case of Vy 2-2 is different. With an integrated intensity of $\sim 48 \mathrm{mJy} \mathrm{km} \mathrm{s}^{-1}$, we have obtained a $6 \sigma$ detection. Only the $F=3-3$ maser line transition lying at $6035 \mathrm{MHz}$ was detected. No absorption or emission can be observed for the other transitions. Figure 4 shows the observed $6035 \mathrm{MHz}$ spectrum. 
Table 2. Gaussian line parameters of the $6035 \mathrm{MHz} \mathrm{OH}$ emission line of Vy 2-2.

\begin{tabular}{ccc}
\hline \hline $\begin{array}{c}\text { Velocity } \\
\left(\mathrm{km} \mathrm{s}^{-1}\right)\end{array}$ & $\begin{array}{c}\text { Peak flux density } \\
(\mathrm{mJy})\end{array}$ & $\begin{array}{c}\text { Linewidth } \\
\left(\mathrm{km} \mathrm{s}^{-1}\right)\end{array}$ \\
\hline$-63.0 \pm 0.14$ & $38 \pm 8$ & $1.14 \pm 0.17$ \\
$-61.6 \pm 0.14$ & $39 \pm 8$ & $0.84 \pm 0.17$ \\
\hline
\end{tabular}

The parameters and uncertainties $(1 \sigma)$ of Gaussian fits to the detected features are displayed in Table 2. The derived apparent luminosity is $1.1 \mathrm{Jy} \mathrm{km} \mathrm{s}{ }^{-1} \mathrm{kpc}^{2}$ (assuming a distance of $3.8 \mathrm{kpc}$, Bensby \& Lundström 2001). The lack of $F=2-2$ emission $^{2}$ and the narrow $F=3-3$ linewidth suggest that the observed $F=3-3$ line results from a maser process. However, only interferometric observation could give a definitive proof of it.

This detection is consistent with the results of Jewell et al. (1985) who observed maser emission at nearly the same velocity $\left(\sim-62.3 \mathrm{~km} \mathrm{~s}^{-1}\right)$ and with about the same line width $\left(\sim 1.5 \mathrm{~km} \mathrm{~s}^{-1}\right)$ but with a peak flux intensity four times stronger $(0.15 \mathrm{Jy})$. The presence of the two features (Fig. 4) is likely real. After splitting our data in two equal parts, the same two components appear. In another data reduction test, we have degraded our spectral resolution. This yields one single feature with a line width of $\sim 2.5 \mathrm{~km} \mathrm{~s}^{-1}$, i.e. twice the line width observed by Jewell et al., centered around $-62.3 \mathrm{~km} \mathrm{~s}^{-1}$. Our observations and data reduction confirm long term $\mathrm{OH}$ emission from Vy 2-2.

\section{3. $V y 2-2$}

As is the case for other Galactic planetary nebulae, the distance to Vy 2-2 (G045.4-02.7) is poorly known. Previous attempts to determine the distance have resulted in a wide range of estimates. Those estimates put this object from $1.9 \mathrm{kpc}$ (see Acker 1978) based on an optical calibration to a kinematic distance of $20 \mathrm{kpc}$ (Davis et al. 1979). The most recent estimate, based on a compilation of previous measurements (see Bensby \& Lundström 2001) gives a distance of $3.8 \mathrm{kpc}$. Vy 2-2 is a source of free-free radio continuum radiation and dust-type infrared emission. VLA maps show a slightly elongated continuum source (Seaquist \& Davis 1983). The continuum emission originates from a compact (diameter $\sim 0.5^{\prime \prime}$ ) and narrow (thickness $\sim<0$.'12) shell of ionized gas. This ionized region is surrounded by an extended halo of over $25^{\prime \prime}$ in radius, detected through its $\mathrm{H} \alpha$ line emission (see Miranda \& Solf 1991). From the visibility analysis, Christianto \& Seaquist (1998) estimate an angular expansion of $1.13 \pm 0.12 \mathrm{mas} / \mathrm{yr}^{-1}$. This would give for a distance of $3.8 \mathrm{kpc}$ an expansion velocity of about $20 \mathrm{~km} \mathrm{~s}^{-1}$, in contradiction with the expansion velocity of $6 \mathrm{~km} \mathrm{~s}^{-1}$ measured by Miranda \& Solf (1991) in the equatorial plane and qualified to be slow. Taking a systemic velocity for the source of $-44.3 \pm 1.0 \mathrm{~km} \mathrm{~s}^{-1}$ (tentative detection of Knapp \& Morris 1985) this would give a blue-shifted velocity

\footnotetext{
2 In the LTE approximation we obtain $F=3-3 / F=2-2$ approx 1.4 whereas we observe here $>2$.
}

for the $\mathrm{OH}$ maser of about $20 \mathrm{~km} \mathrm{~s}^{-1}$. The inferred expansion velocity is then in good agreement with the value derived by Christianto \& Seaquist (1998) for a distance of $3.8 \mathrm{kpc}$. The kinematic age of the nebula they derived is 213 years and supports the conclusion that this object is a very young planetary nebula. The temperature of the central star is estimated to be greater than $35000 \mathrm{~K}$ (see Zijlstra et al. 1989; Clegg \& Walsh 1989). The dust color temperature was estimated by Cohen $\&$ Barlow (1974) to be less than 190K.

Jewell et al. (1985) and Cohen et al. (1991) searched without success for ${ }^{2} \Pi_{1 / 2}, J=1 / 2$ maser emission (down to a $3 \sigma$ limit of $\sim 0.25 \mathrm{Jy}$ ). The $1612 \mathrm{MHz}$ maser emission, the only ground-state maser transition observed, was first detected by Davis et al. (1979). Seaquist \& Davis (1983) located the maser at the front edge of the ionized shell, coincident with a shock front and an ionization front, placing the $\mathrm{OH}$ maser on the near side of the expanding shell and thus providing an explanation for the blue-shifted maser feature. This is consistent with the fact that $\mathrm{OH}$ molecules are effectively produced in the outer parts of circumstellar envelopes due to photoionization of $\mathrm{H}_{2} \mathrm{O}$ by interstellar UV photons. The typical abundance for $\mathrm{OH}$ molecules relative to $\mathrm{H}_{2}$ is about $10^{-5}$ and HST observations (see Sahai \& Trauger 1998) show a compact bright bipolar source expanding along an axis roughly orthogonal to the bipolar axis. Despite the fact that almost all planetary nebulae appear optically thin at $5 \mathrm{GHz}, \mathrm{Vy} 2-2$ is optically thick (see Purton et al. 1982).

\section{Implications on pumping schemes}

There exist two main ways to invert the $5 \mathrm{~cm}$ maser transition, (1) by radiative pumping of far infrared (FIR) photons or (2) by collisions with $\mathrm{H}_{2}$ molecules (in combination with local and non-local line overlap). Chemical pumping does not seem applicable and interstellar UV photons are only responsible for the dissociation of $\mathrm{H}_{2} \mathrm{O}$ molecules in the outer part of the circumstellar envelope to produce $\mathrm{OH}$ (the contribution of stellar UV photons is negligible except perhaps for the inner regions of the envelope).

\subsection{About the ground-state masers}

Theoretical studies of the pumping mechanism of the $18 \mathrm{~cm}$ $\mathrm{OH}$ lines are quite advanced. The absorption of FIR photons at $34.6 \mu \mathrm{m}$ and $53.3 \mu \mathrm{m}$ excites the $\mathrm{OH}$ from the ground state to the ${ }^{2} \Pi_{1 / 2}$ ladder. Subsequent cascading of the populations through the $J=1 / 2$ and $J=3 / 2$ levels inverts the $J=3 / 2$ ground state (Elitzur et al. 1976). This scheme explains rather well the strong $1612 \mathrm{MHz}$ line and essentially avoids the ${ }^{2} \Pi_{3 / 2}$ ladder (Davis et al. 1979). Therefore, in circumstellar regions where $1612 \mathrm{MHz}$ is strong and the above mechanism prevails one should not expect to detect $5 \mathrm{~cm} \mathrm{OH}$ emission.

However, the pump cycle may differ in inner layers where highly excited lines are expected to be found. Moreover, Collison \& Nedoluha $(1994,1995)$ argue that FIR line overlaps are also important to enhance the $1612 \mathrm{MHz}$ line and may even be the primary inverting scheme for the $1612 \mathrm{MHz}$ line in not too optically thick $\mathrm{OH}$ envelopes. In the latter case 


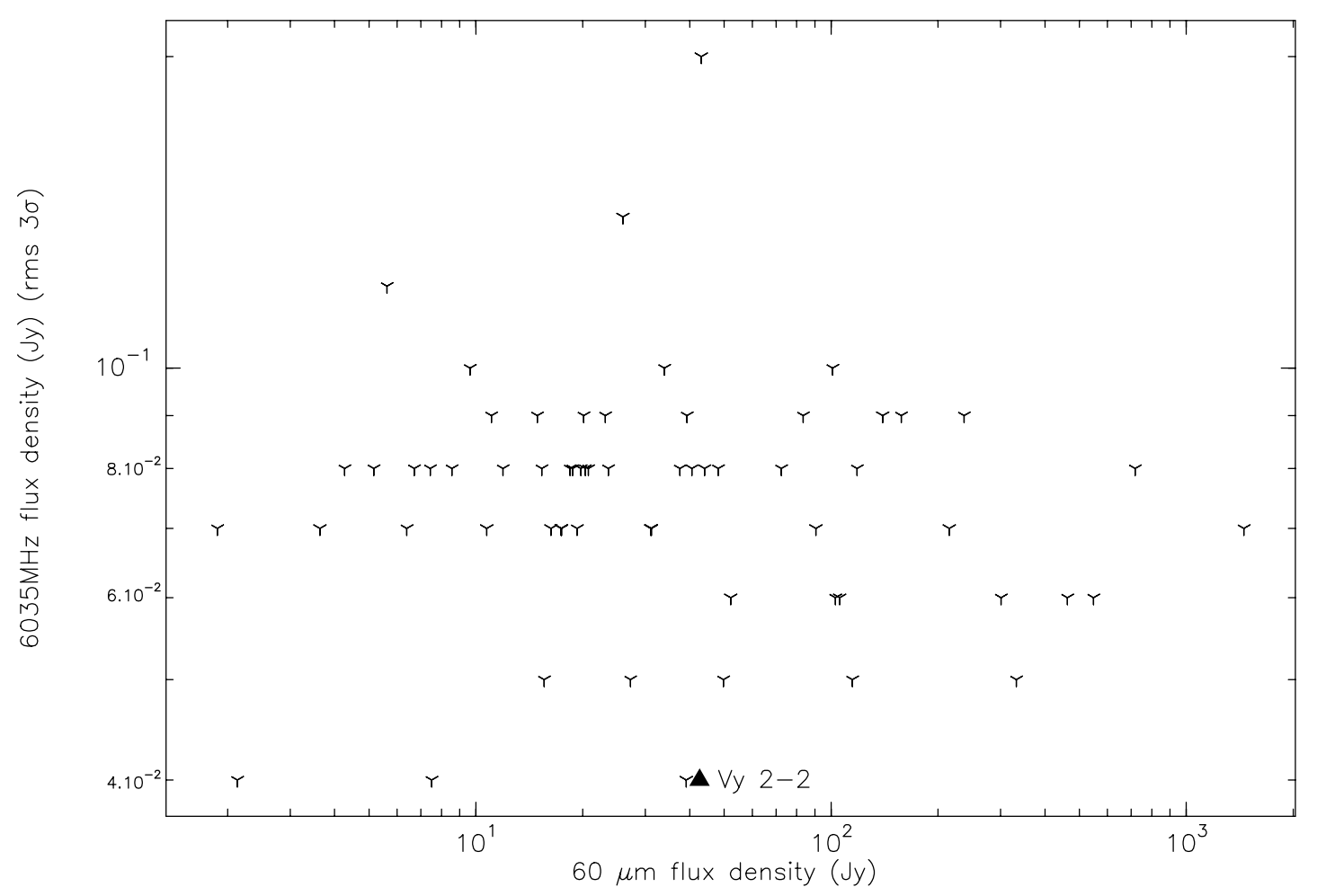

Fig. 5. IRAS $60 \mu \mathrm{m}$ versus the $\mathrm{OH} 6035 \mathrm{MHz} 3 \sigma$ flux density limit (channel $0.25 \mathrm{~km} \mathrm{~s}^{-1}$ ). The filled triangle corresponds to the only detection in our sample, Vy $2-2$, the detected flux is given.

overlaps at $120 \mu \mathrm{m}$ are important and populating the $J=$ $5 / 2$ level is essential. Collison \& Nedoluha (1994) argue that FIR line overlaps alone cannot explain the main-line masers in stars (contrary to earlier models) and that near infrared (NIR) overlap effects (with $\mathrm{OH}$ or $\mathrm{H}_{2} \mathrm{O}$ ) are likely needed to explain the main line emission from thin circumstellar shells.

The most recent model published on $\mathrm{OH}$ masers in circumstellar envelopes (see Thai-Q-Tung et al. 1998) treats only the ground-state excitation and considers two models. The first one is with line overlapping limited by a Doppler shift of $2 \mathrm{~km} \mathrm{~s}^{-1}$ and the other one with large overlapping (up to the expansion velocity). In both models the $1612 \mathrm{MHz}$ appears much stronger than the other ground state lines by a factor $10^{2}$ to $10^{3}$. They found that the pumping based on FIR hyperfine line overlapping is much smaller in the second case. They suggest that FIR line overlapping occurs inside clumps (small Doppler shift) of circumstellar envelopes (this idea was also invoked by Collison and Nedoluha), but no prediction is made about the excited state. Only the recent work of Pavlakis \& Kylafis (1996, 2000) studied excited OH maser emission but only in the case of massive star forming regions.

Modeling the detected maser emission in Vy 2-2 at 1612 and $6035 \mathrm{MHz}$ is a challenge. The particular nature of the source, a very young proto planetary nebulae, may certainly be a clue. The fact that two masers are observed at the same LSR velocity $\left(-62 \mathrm{~km} \mathrm{~s}^{-1}\right)$ argues in favor of their spatial association. In such a case they would both originate from the thin ionization shell presenting similar conditions as in HII regions. Within this context, PPN shells may be characterized by particularly high densities and long path lengths for coherent amplification that have to be taken into account.

\subsection{Infrared pumping?}

Excitation of the $\mathrm{OH}$ radical results from complex competitive schemes involving both collisional and radiative pumping as well as line overlap effects that are correlated with the velocity field in the $\mathrm{OH}$ medium and local line broadening. The $5 \mathrm{~cm}$ $\mathrm{OH}$ lines arise from energy levels $84 \mathrm{~cm}^{-1}$ above the groundstate and we therefore expect that FIR photons around $100 \mu \mathrm{m}$ are involved in the $\mathrm{OH}$ pumping cycle. To evaluate the possibility of a pumping scheme based only on IR photons, Fig. 5 compares the IRAS flux at $60 \mu \mathrm{m}$ and the lower limits of $\mathrm{OH}$ emission at $6 \mathrm{GHz}$, assuming that the ratio between the radio solid angle and the IR solid angle is $\approx 1$. Figure 5 shows that the number of FIR photons largely exceeds the emitted radio photons. Baudry et al. (1997) reached a similar conclusion for compact HII regions but in that case many $5 \mathrm{~cm} \mathrm{OH}$ masers could be detected. From this we conclude that in stellar envelopes the $\mathrm{OH}$ pumping mechanism is different from that in massive star forming regions and that the available FIR radiation is unlikely to work as a pump for this maser. Moreover as the envelopes are dense, it is possible that even if the IR pumping were efficient, collisions could effectively quench the $\mathrm{OH}$ maser emission.

\section{Conclusion}

We have observed an extensive sample of OH/IR stars and late-type variables. Except for one atypical source (Vy 2-2) no excited $\mathrm{OH}$ maser has been detected. We do not confirm 
the tentative detection of NML Cyg. Only the blue shifted emission is detected in Vy 2-2 for which we have discussed briefly the morphology and $\mathrm{OH}$ production. The absence of detectable excited emission at $5 \mathrm{~cm}$ (except the unique object Vy 2-2) tends to argue in favor of a pumping scheme based essentially on the absorption of 35 and $53 \mu \mathrm{m}$ photons. In the case of Vy 2-2 the ionization shell from where the maser emission seems to originate, may present physical conditions (shock, higher temperature and density) similar to those prevailing in HII regions. A hybrid pumping model applying to both $\mathrm{OH} / \mathrm{IR}$ stars and HII regions or even a pumping scheme similar to $\mathrm{OH}$ maser emission in massive star forming regions may be successful. It is interesting to note that no absorption at 35 and $53 \mu \mathrm{m}$ can be seen for this source in ISO observations. Interferometric observations are needed to spatially determine the likely related positions of the 1612 and $6035 \mathrm{MHz}$ maser emission in Vy 2-2.

Acknowledgements. This research has made use of the SIMBAD database operated at CDS, Strasbourg, France and ASTRID database, operated at GRAAL, Montpellier, France. We thank Dr A. M. S. Richard for valuable suggestions to prepare the observations and N. J. Rodríguez-Fernández for his help on ISO data.

\section{References}

Acker, A. 1978, A\&AS, 33, 367

Baudry, A. 1974, A\&A, 32, 191

Baudry, A., Desmurs, J.-F., Wilson, T. L., \& Cohen, R. J. 1997, A\&A, 325,255

Bensby, T., \& Lundström, I. 2001, A\&A, 374, 599

Cesaroni, R., \& Walmsley, M. C. 1991, A\&A, 241, 537

Christianto, H., \& Seaquist, E. R. 1998, AJ, 115, 2466

Claussen, M. J., \& Fix, J. D. 1981, ApJ, 250, L77

Clegg, R., \& Walsh, J. R. 1989, IAU, 131, p443

Cohen, M., \& Barlow, M. J. 1974, ApJ, 193, 401
Cohen, M., Masheder, M. R. W., \& Walker, R. N. F. 1991, MNRAS, 250,611

Collison, A. J., \& Nedoluha, G. E. 1994, ApJ, 422, 193

Collison, A. J., \& Nedoluha, G. E. 1995, ApJ, 442, 311

David, P., Le Squeren, A. M., \& Sivagnanam, P. 1993, A\&A, 277, 453

Davis, L. E., Seaquist, E. R., \& Purton, C. R. 1979, ApJ, 230, 434

Elitzur, M., Goldreich, P., \& Scoville, N. 1976, ApJ, 205, 384

Engels, D. 1979, A\&AS, 36, 337

Epchtein, N., Guibert, J., Nguyen-Quang-Rieu, Mr., Turon, P., \& Wamsteker, W. 1980, A\&A, 85, L1

Gray, M. D., Field, D., \& Doel, R. C. 1992, A\&A, 262, 555

Jewell, P. R., Schenewerk, M. S., \& Snyder, L. E. 1985, ApJ, 295, 183

Knapp, G. R., \& Morris, M. 1985, ApJ, 292, 640

Miranda, L. F., \& Solf, J. 1991, A\&A, 252, 331

Moore, T. J. T., Cohen, R. J., \& Mountain, C. M. 1988, MNRAS, 231, 887

Ott, M., Witzel, A., Quirrenbach, A., et al. 1994, A\&A, 284, 331

Pavlakis, K. G., \& Kylafis, N. D. 1996, ApJ, 467, 300

Pavlakis, K. G., \& Kylafis, N. D. 1996, ApJ, 467, 309

Pavlakis, K. G., \& Kylafis, N. D. 2000, ApJ, 534, 770

Purton, C. R., Feldman, P. A., Marsh, K. A., Allen, D. A., \& Wright, A. E. 1982, MNRAS, 198, 321

Sahai, R., \& Trauger, J. T. 1998, AJ, 116, 1357

Seaquist, E. R., \& Davis, L. E. 1983, ApJ, 274, 659

Sivagnanam, P., Le Squeren, A. M., \& Foy, F. 1988, A\&A, 206, 285

Sylvester, R. J., Barlow, M. J., Liu, X. W., et al. 1997, MNRAS, 291, L42

Thacker, D. L., Wilson, W. J., \& Barrett, A. H. 1970, ApJ, 161, L191

Thai-Q-Tung, Dinh-V-Trung, Nguyen-Q-Rieu, et al. 1998, A\&A, 331, 317

Yen, J. L., Zuckerman, B., Palmer, P., \& Penfield, H. 1969, ApJ, 156, L27

Zijlstra, A. A., Te Lintel Hekkert, P., Pottasch, S. R., et al. 1989, A\&A, 217,157

Zuckerman, B., Palmer, P., Penfield, H., \& Lilley, A. E. 1968, ApJ, 153, L69

Zuckerman, B., Yen, J. L., Gottlieb, C. A., \& Palmer, P. 1972, ApJ, 177,59 